\title{
High Quality Multimedia Content Sharing Based on Network Coding Over Multiuser Cognitive Networks
}

\author{
Zhang $\mathrm{Yi}^{1}$, Xiao $\mathrm{Shi}^{1}$, Li Jin ${ }^{1}$, Wang Jun ${ }^{1}, \mathrm{Xia} \mathrm{Ge}^{1}$ and Wang Zhujuan ${ }^{2}$ \\ ${ }^{1}$ School of Electronic \& Electrical Engineering, Wuhan Textile University, Wuhan \\ 430020, China \\ ${ }^{2}$ Wuhan Research Institute of Post and Telecommunications, Wuhan 430074,
} China

\begin{abstract}
According to the recently published report by the Federal Communications Commission, the spectrum regulatory authority in the United States, traffic-dist ibution across the radio spectrum is extremely uneven. The phenomenon can be utilized to improve the multimedia content sharing. This paper proposes a novel model of multimedia sharing in wireless communication network. The proposed multimedia sharing mechanism offer services to users who want to access the multimedia networks. At the same time, it does not affect the QoS of existing paying user. The numerical results show that the proposed sharing mechanism provides higher throughput and quality of service than the existing system.

Keywords: multimedia content sharing, QoS, continupus time Markov chain, failing probabilities

\section{Introduction}

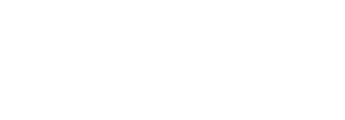

As a program of entertainment and passing the time, video/audio program is certainly a good choice. Some consunets wish to access the multimedia network freely, that is to say, they want to enjoy without paying (called unlicensed user or non-paying user). Nonpaying users can bear progran's discontinuity and advertisement's occurring frequently, because they do not pay for it. As the video playing needs waiting for a long time (20 seconds or more), these users would choose to give up these programs, which causes the advertising push and relay transport process to fail. The purpose of this paper is to design a multimedia sharing mechanism, which can make non-paying users enjoy better payment services, at the same time, without affecting the QoS of existing paying users.

Existing spectrum regulation has advantages, for example, it is easy to manage. But there are also disadvantages, for example, spectrum resources cannot meet the needs of users. Ao the demand for spectrum resource increases rapidly (e.g., [1-3]), there are increasingly more challenges and complications yet ironed out. For example, the application of a large portion of the assigned wireless spectrum is uneven [4], leading to underutilization of a significant amount of spectrum. According to the published report by the Federal Communications Commission (FCC), unlicensed portions of the spectrum (e.g. $2.4 \mathrm{GHz}$ and $5 \mathrm{GHz}$ bands) are heavily occupied by most wireless networks operate (e.g. WLAN and $\mathrm{WiFi}$ ), whereas the licensed portions of the spectrum (e.g. TV White Space band) are used sporadically. Hence, dynamic spectrum access (DSA) techniques were proposed to solve these spectrum inefficiency problems in which the non-paying users can exploit unused portions of the licensed spectrum while safeguarding the transmissions of the paying users. The sharing scheme provides the capability to share the wireless spectrum resource with non-paying users in an opportunistic operation. On the one hand, robust CR can effectively suppress interference to nearby licensed users in [5].
\end{abstract}


On the other hand, [6] study the interaction between CR and Digital TV. The images are shown on video graphics array monitor in real time [21]. The energy effective MAC protocol is superior in improving throughput and multimedia transmission delay [22]. Video multicast and video conferencing systems in WLAN are studied in [23].

For the non-paying users, the spectrum availability varies as the licensed user's access. This characteristic complicates coordination among the non-paying users during data transmission. In order to cope with the characteristic, non-paying users achieve several important functions. Some aspects have been studied well (e.g., spectrum sensing) [7-15]. However, there are a lot of problems to tackle in sharing.

It is due to the better sharing scheme can improve the User Experience (UE) and network performance greatly, so scholars actively study the correlative technology and many excellent mechanism are emerging in the past ten years. Compared with the optimal model and competitive model, the cooperative model [16] could reach the maximum total profit for unlicensed users with better fairness. A modified 802.11-based opportunistic spectrum access is proposed for single-channel wireless networks where licensed users operate on a slot-by-slot basis. Continuous-time Markov chain was used to de veloped analytical model and derive the expressions to compute the grade of service performance metrics. Buffering mechanism is proposed for the unlicensed subscriber. Prioritized unlicensed user traffic (i.e., high transmission rate and low transmission rate) is considered [17-21].

Unlike the above works, in this paper, we consider prioritization among the video traffic and text traffic of non-paying users while accessing the licensed spectrum. For example, the unlicensed users with video traffic have higher priority than those with text traffic. Further, text traffic are going to be operated with hetwork coding if there is no enough channel to access. We use continuous-time Markov chain to analyze the model. The performance of the model is evaluated in terms of the general parameters, such as blocking probability of video and ext traffic, the termination probability of video and text traffic, and the system throughput includingoth video traffic and text traffic of nonpaying users'.

\section{Proposed System Model}

The proposed system model shown in Figure 1 consists of a licensed user network and an unlicensed user network. A licensed user network, operating over a given service area, contains a licensed base station and licensed users. Then, an unlicensed user network contains an unlicensed base station and non-paying users. The wireless spectrum is shared by the paying users and non-paying users. The paying users have the highest priority in using the channels. The traffic of non-paying users are classified into two priority classes. The high priority hon-paying user are denoted as video traffic user (VU) while the low priority non paying user are denoted as text traffic user (TU). On one hand, the licensed service area DI can be extended to D2 by TU1 (i.e., relay link C2). On the other hand, licensed user LU2 obtain better signal quality and higher transmission rate through relay link C3. C4 and C5. 


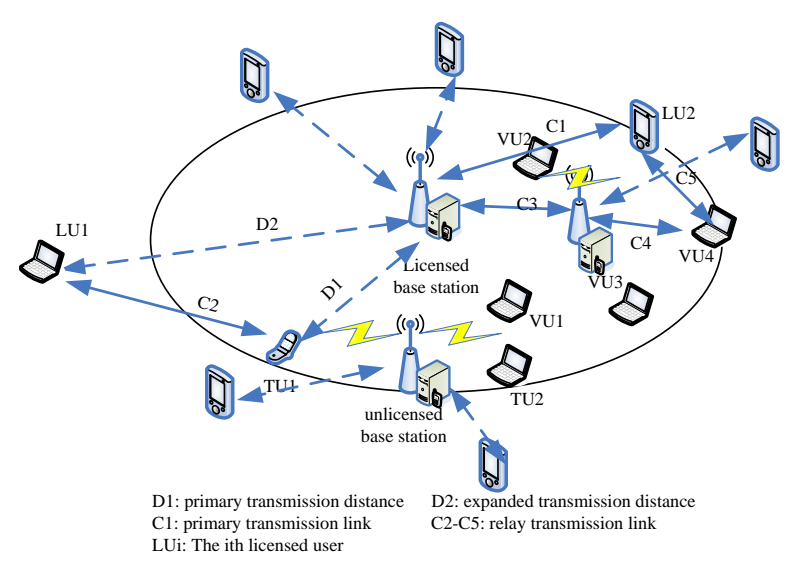

\section{Figure 1. System Model}

The system model of proposed sharing mechanism is presented in Figure 2 , where a higher priority is given to video traffic over text traffic. For non-paying asers, text traffic is replaced with video traffic to access channel when only one channel Is idle. Both video traffic and text traffic of SU are going to be dropped hen all channels are occupied by licensed users. Otherwise, the text traffic of non-paying user can be transmitted by means of coding. A licensed user will be blocked (blocked licensed user rraffic) if all wireless resource are occupied by other licensed user. If all channels are busy and a licensed user call arrive, non-paying user will be terminated (terminated non paying user traffic).

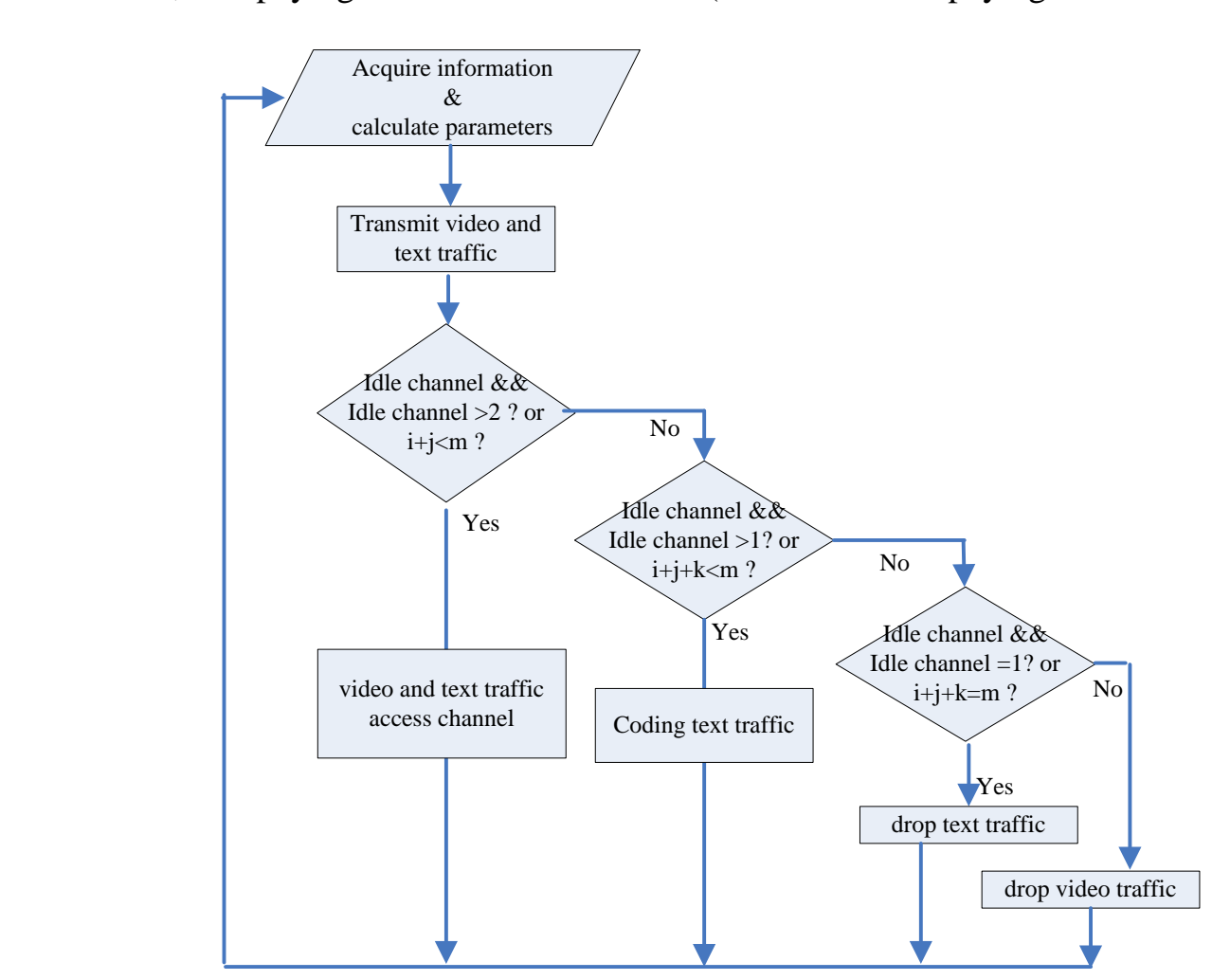

Figure 2. Proposed Users Sharing Mechanism 


\section{Steady State Performance Analysis}

In this section, we develop the model using continuous time Markov chain (CTMC). The state of the CTMC is defined as $(i, j, k)$, where $i \in(0,1,2, \cdots, m), j \in(0,1,2, \cdots, m)$ and $k \in(0,1,2, \cdots, m)$ represent the number of ongoing licensed user, video traffic of non-paying user and text traffic of non-paying user in the scheme respectively. A valid state $(i, j, k)$ should not exceed $m$.

Table 1. System Parameters

\begin{tabular}{|c|c|}
\hline$m$ & The number of all licensed channels \\
\hline$i$ & The number of ongoing PU, $i \in(0,1,2, \cdots, m)$ \\
\hline$j$ & The number of ongoing video traffic of SU, $j \in(0,1,2, \cdots, m)$ \\
\hline$k$ & The number of ongoing text traffic of SU, $k \in(0,1,2, \cdots, m)$ \\
\hline$g_{i j}$ & transition probability from state $i$ to tate $j$ \\
\hline$\Psi$ & The state space \\
\hline$\lambda$ & Arrival ate \\
\hline$\mu$ & The mean of service times \\
\hline$\pi_{i, k, j}$ & The steady state probability for a state $i, j, k)$ \\
\hline
\end{tabular}

The state of the Markov chain model is defined as $\Psi=(i, j, k)$. We assume that the licensed user, video traffic of nor-paying user and text traffic of non-paying user arrival processes follow Poisson process with the arrival rate $\lambda_{1}, \lambda_{2}$ and $\lambda_{3}$, respectively. The service times for them follow an exponential distribution with mean $1 / \mu_{1}, 1 / \mu_{2}$ and ${ }_{1 /}{ }^{\mu}{ }_{3}$, respectively. Let $(i, j, k$ ) represent a system state, where $i, j, k$ denote the number of channels used by the licensed user, video traffic of non-paying user and text traffic of nonpaying user, respectively. The system state transitions in $\Psi$ can be depicted in Figure3.

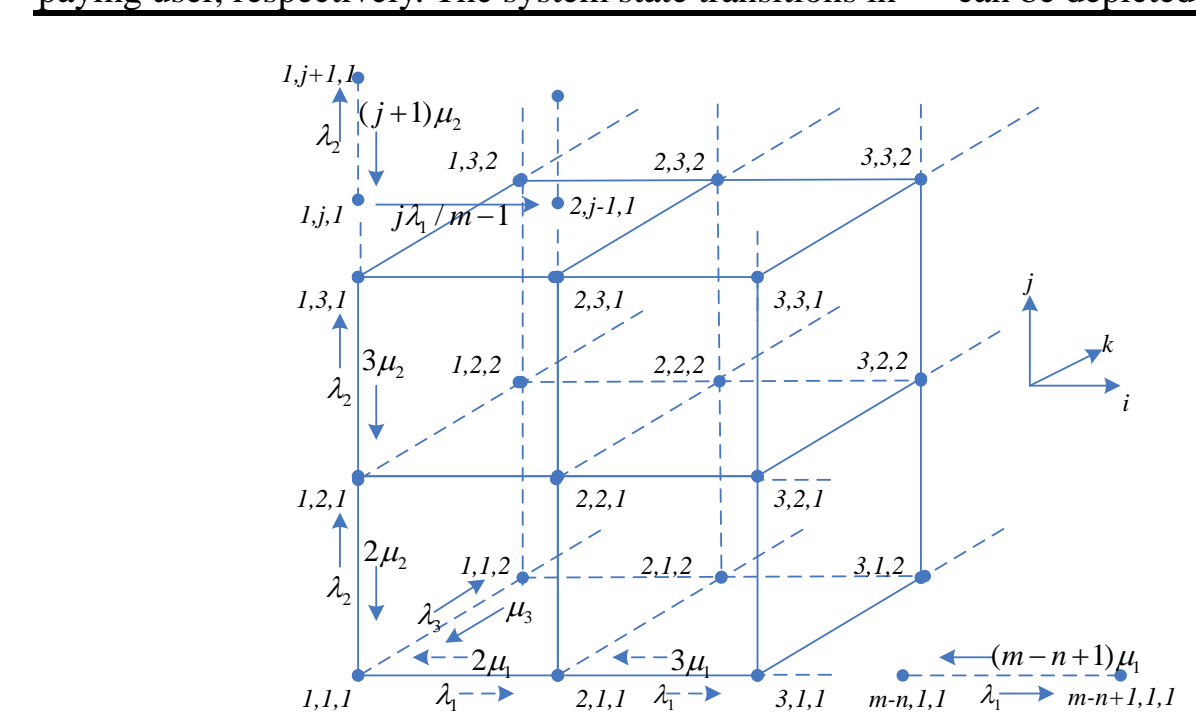

Figure 3. System State Transitions Diagram in 3-Dimension 
The evolution of the state $(i, j, k)$ of the Markov process is presented under three cases:

$$
\begin{gathered}
i+j<m, k=0 \\
i+j+k<m, k \neq 0 \\
i+j+k=m
\end{gathered}
$$

According to dynamic multimedia sharing scheme of system presented above, the system state transitions diagram can be obtained as in Figure3. On the basis of the transition rate diagram, we can get the set of global balance equations as following.

Objective:

$$
\left\{\prod \mid \pi_{1,} \pi_{2} \pi_{3,} \cdots, \pi\right\}
$$

s.t.

$$
\begin{aligned}
& \sum_{j} \pi_{i} g_{i j}=\sum_{i} \pi_{j} g_{j i} \\
& i \mu_{1} \pi_{(i, j, 0)}-\lambda_{1} \pi_{(i-1, j, 0)}+j \mu_{2} \pi_{(i, j, 0)}-\lambda_{2} \pi_{(i, j-1,0)} \\
& +\lambda_{1} \pi_{(i, j, 0)}-(i+1) \mu_{1} \pi_{(i+1, j, 0)}+\lambda_{3} \pi_{(i, j, 0)} \\
& -\mu_{3} \pi_{(i, j, 1)}+\lambda_{2} \pi_{(i, j, 0)}-(j+1) \mu_{2} \pi_{(i, j+1,0)}=0 \\
& i \mu_{1} \pi_{(i, j, k)}-\lambda_{1} \pi_{(i-1, j, k)}+j \mu_{2} \pi_{(i, j)} \\
& -\lambda_{2} \pi_{(i, j-1, k)}+k \mu_{3} \pi_{(i, j, k)}-\lambda \pi_{(i, j, k-1)} \\
& +k \lambda_{1} /(m-i) \pi_{(i, j, k)}-\mu_{1} \lambda_{3} \pi_{(i+1, j, k-1)} \\
& +j \lambda_{1} /(m-i) \pi_{(i, j, j)} \mu_{1} \lambda_{2} \pi_{(i+1, j-1, k)}=0 \\
& \left.\pi_{(i, j, k)}=0,(i, j, k) \mid(i, \hat{\jmath}, k \notin \psi\rangle\right\} \\
& \Delta \sum_{j} \sum_{k} \pi_{(i, j, k)}=1
\end{aligned}
$$

While, the total number of occupied channels in the state $(i, j, k)$ should satisfy the following condition:

$$
\Psi=\{(i, j, k) \mid i+j+k \leq m\}
$$

where $g_{i j}$ denotes transition probability from state $i$ to state $j . \pi_{(i, j, k)}$ denotes the steady state probability for a state $(i, j, k)$. Then, Combining equations above and solving the linear program (5) $-(10)$, we can get $\left(\pi_{1}, \pi_{2}, \pi_{3}, \cdots, \pi_{l}\right)$. The array can be calculated with the flow shownas.

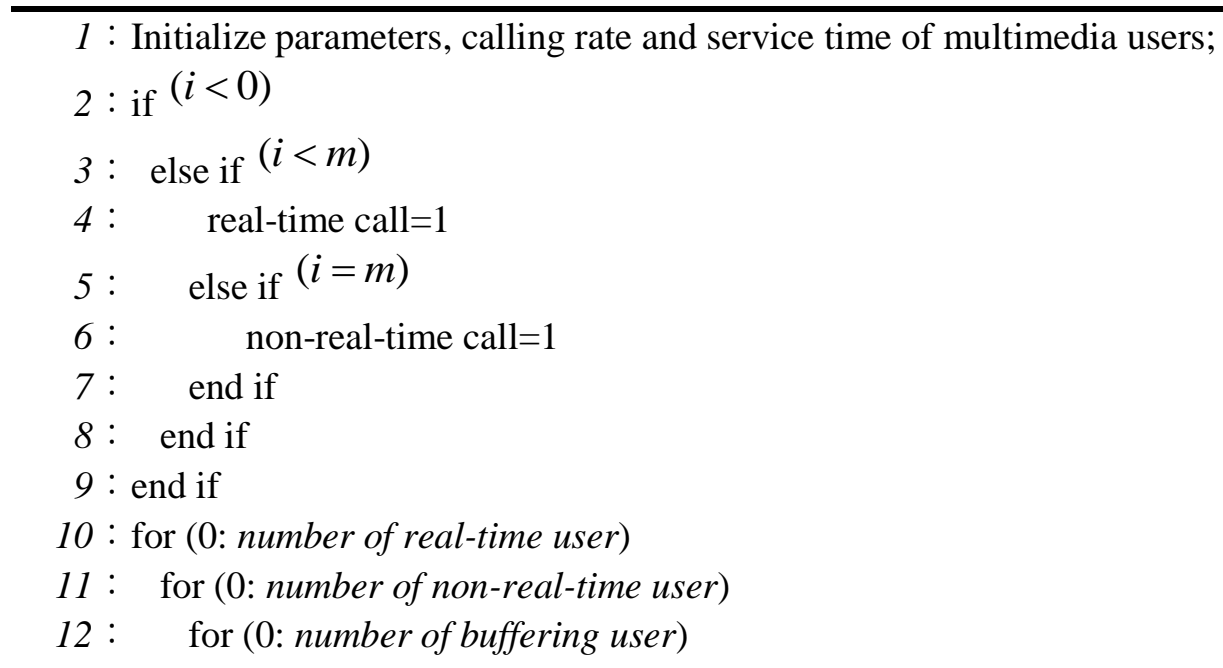


13: $\quad$ calculate steady states;

$14:$

$$
\Pi=\left(\begin{array}{ccc}
\pi_{0}^{1} & \pi_{0}^{2} & \pi_{0}^{3} \\
\vdots & \vdots & \vdots \\
\pi_{i}^{1} & \pi_{j}^{1} & \pi_{k}^{1}
\end{array}\right)
$$

$15:$ seek matrix, len $=$ state space;

$16:$ for $(0: l e n)$

17 : calculate $\Psi$;

\section{Performance Evaluation}

Based on the steady state probability analysis, QoS performance metric can be calculated, including system throughput, termination probability and blocking probability. The metric is expressed by function $Q \sim\left[T h r, P_{\text {termination, }}, P_{b l o c k}\right]$, where $T h r, P_{\text {terminaion }}$ and $P_{\text {block }}$ are denoted as throughput, termination probability and blocking probability, respectively.

For the video traffic of non-paying user, it may be interupted by the arrival of a licensed user call when no channel is idle. Let Ptemmination_video denote interruption probability of video traffic. So $P_{\text {termination_video }}$ can be obtained as

$$
P_{\text {termination_video }}=\frac{\sum_{(i, j, k) \in \Psi \mid k=0} \pi_{i, j, k}}{\sum_{, k, \in) \in \mid j>0} \pi_{i, j, k}}
$$

that a channet 6 coupied Then, the probability that a channet occupied oy the coded text traffic is reclaimed by a $\mathrm{PU}$ is $\mathrm{k} /(m-i)$. For the text traffic Det $P_{\text {termination_text denote interruption probability of text }}$ traffic. So $P_{\text {termination_text }}$ can be obtained as

$$
2 P_{\text {termination_text }}=\frac{\sum_{(i, j, k) \in \Psi \mid k=0} \frac{k}{m-i} \pi_{i, j, k}}{\sum_{(i, j, k) \in \Psi \mid} \pi_{i, j, k}}
$$

Video traffic and coded text traffic will be blocked when no channel is idle. Let $P_{\text {block_video }}$ and $P_{\text {block_text }}$ are denoted as blocking probability of video traffic and text traffic, respectively. So blocking probability for video traffic and text traffic can be calculated, respectively, as

$$
\begin{gathered}
P_{\text {block_video }}=\sum_{(i, j, k) \in \Psi \mid i+j+k=m, k=0} \pi_{i, j, k} \\
P_{\text {block_text }}=\sum_{(i, j, k) \in \Psi \mid i+j+k=m} \pi_{i, j, k}
\end{gathered}
$$

LeT Thr denote the total traffic load of SU. Thus, the throughput can be calculated as

$$
T h r=\sum_{(i, j, k) \in \Psi \mid m} \pi_{i, j, k}\left(\sum_{(i, j, k) \in \Psi \mid j=1}^{m} \pi_{i, j, k} \mu_{1}+\sum_{(i, j, k) \in \Psi \mid k=1}^{m} \pi_{i, j, k} \mu_{2}\right)
$$

Further, the QoS mentioned above can be given.

\section{Numerical Results}

After description of the sharing scheme implementation of the non paying users, we present numerical results by comparing the proposed scheme with general scheme. For example, let HandOff-1 denote the method with fair scheme, HandOff-2 denote the method with priority scheme (e.g., video traffic and text traffic for non-paying user), and 
HandOff-3 denote the scheme proposed. In the experiments, we set $\mathrm{m}=5, \lambda_{3} \in[0.01,0.1]$,

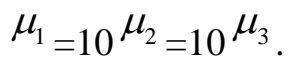

As shown in Figure 4 and Figure 5, the proposed scheme can provide lower termination probability and blocking probability than others for non-paying users. In Figure 6, the analysis result of system traffic load is given. With the rapidly increasing of data arrival rate, the proposed handoff scheme can support the largest system load. In Figure 7, we can find that the proposed handoff scheme can achieve the best performance.

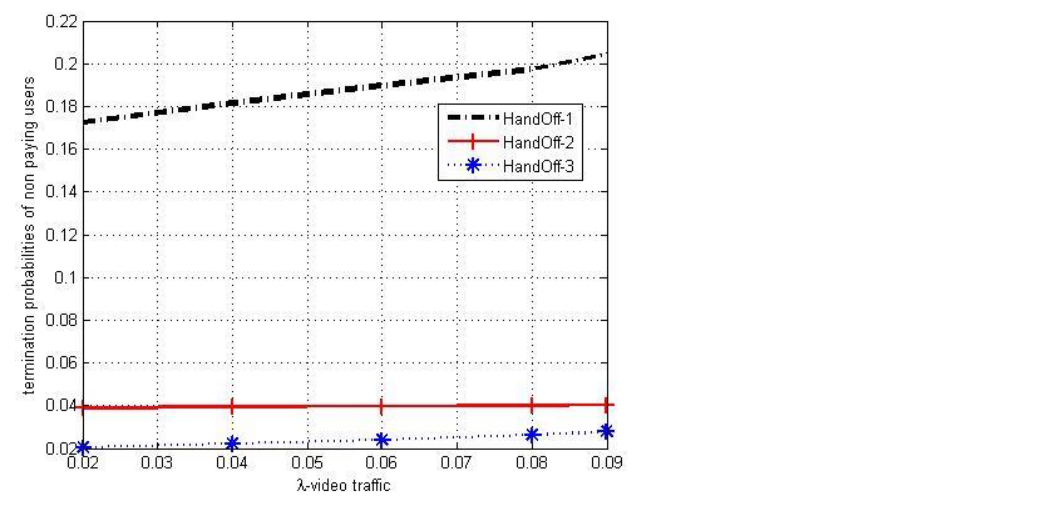

Figure 4. Comparison of Termination Probabilities with Different Handoff Schemes

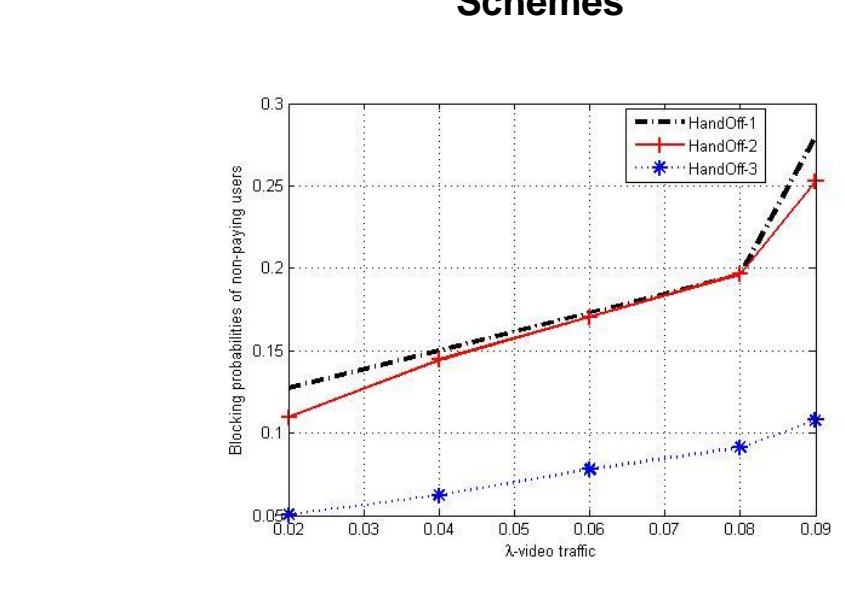

Figure 5. Comparison of Blocking Probabilities with Different Handoff

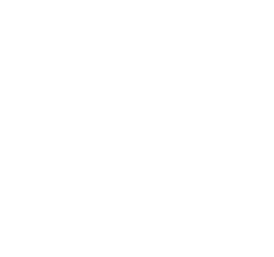

Schemes

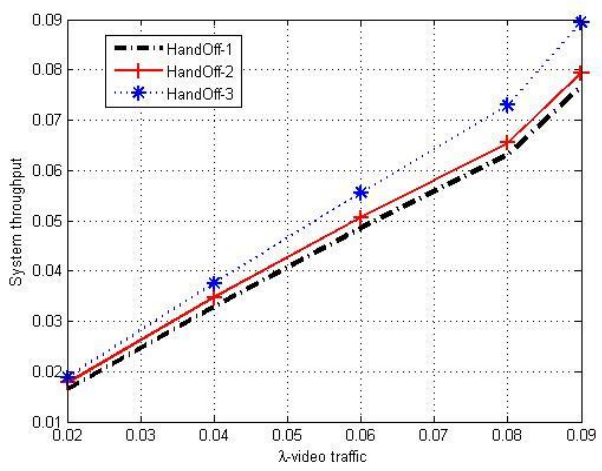

Figure 6. Comparison of System Throughput 


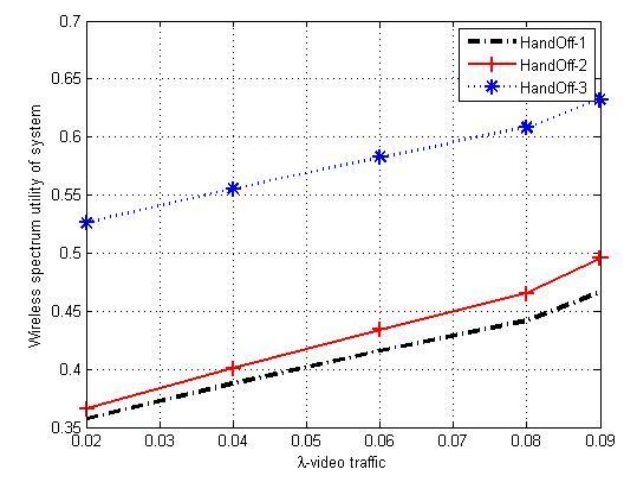

Figure 7. Comparison of System Spectrum Resource Utility

\section{Conclusion}

In this paper, we proposed a novel model of multimedia sharing in wireles network and analyze how to improve the non-paying users' QoS tmough mathematical method. Numerical results also showed that the proposed handoff seheme can effectively match the theoretical analysis.

\section{Acknowledgement}

Funds of Wuhan Textile University (155028)

\section{References}

[1] K. Yun, K. Kim, N. Hur, S. I. Lee and G. H. Park, "Elficient Multiplexing Scheme of Stereoscopic Video Sequences for Digital Broadcasting Services", TTRI Journal, vol. 32, no. 6, (2010).

[2] S. K. Kim, J. Jeong, H. G. Kim and M. G. Chang, "A Personal Videocasting System with Intelligent TV Browsing for a Practical 1deo Application Environment", ETRI Journal, vol. 31, no. 1, (2009).

[3] C. S. Leem, S. W. Kim C. Kim, S. C. Kang and J. Lee, "Spectral Efficiency of WRAN Spectrum Overlay in the TV White Space", ETRI Journal, vol. 30, no. 6, (2008).

[4] "FCC, ET Docket No 03-322 Notice of Proposed Rule Making and Order", (2003).

[5] N. M. Kim, M. R. Kim, E. J. Kim S. J. Shin, H. I. Yu and S. B. Yun, "Robust Cognitive-Radio-Based OFDM Architecture with Adaptive Traffic Allocation in Time and Frequency", ETRI Journal, vol. 30, no. $1,(2008)$.

[6] Y. K. Yoon, H. J. Hong and I. G. Choi, "Protection of Digital TV from Cognitive Radio Interference", ETRI Journal, vol. 29 no. 3, (2007).

[7] B. Shen and K. S. Kwak, "Soft Combination Schemes for Cooperative Spectrum Sensing in Cognitive Radio Networks", ETRI Journal, vol. 31, no. 3, (2009).

[8] B. H. Kim and K. G. Shin, "Efficient discovery of spectrum opportunities with MAC-layer sensing in cognitive radio networks", IEEE Transactions on Mobile Computing, vol. 7, no. 5, (2008).

[9] Q. Zhao and B. M. Sadler, "A Survey of Dynamic Spectrum Access", IEEE Signal Processing Magazine, vol. 24, no. 3, (2007).

[10] Y. Zeng, Y. C. Liang and T. H. Pham, "Spectrum sensing for OFDM signals using pilot induced autocorrelations", IEEE Journal on Selected Areas in Communications, vol. 31, no. 3, (2013).

[1] W. Zhang, R. K. Mallik and K. Letaief, "Cooperative spectrum sensing optimization in cognitive radio networks", Proceedings of IEEE International Conference on Communications, Beijing, China, (2008).

[12] Z. Quan, S. Cui and A. H. Sayed, "Optimal linear cooperation for spectrum sensing in cognitive radio networks", IEEE Journal of Selected Topics in Signal Processing, vol. 2, no. 1, (2008).

[13] W. Zhang and K. Letaief, "Cooperative spectrum sensing with transmit and relay diversity in cognitive radio networks", IEEE Transactions on Wireless Communications, vol. 7, no. 12, (2008).

[14] G. Ganesan and Y. Li, "Cooperative spectrum sensing in cognitive radio, part I: Two user networks", IEEE Transactions on Wireless Communications, vol. 6, no. 6, (2007).

[15] X. Wang, W. Chen and Z. Cao, "Partially observable Markov decision process-based MAC-layer sensing optimization for cognitive radios exploiting rate less-coded spectrum aggregation", IET Communications, vol. 6, no. 8, (2012).

[16] J. Chen, X. Zhang and Y. Kuo, "Adaptive Cooperative Spectrum Sharing Based on Fairness and Total 
Profit in Cognitive Radio Networks", ETRI Journal, vol. 32, no. 4, (2010).

[17] L. Zhai, and X. Zhang, "Modified 802.11-Based Opportunistic Spectrum Access in Cognitive Radio Networks", ETRI Journal, vol. 34, no. 2, (2012).

[18] T. Luo, F. Lin, T. Jiang, M. Guizani and W. Chen, "Multicarrier Modulation and Cooperative Communication In Multi-hop Cognitive Radio Networks", IEEE Wireless Communication Mag., vol. 18, no. 1, (2011).

[19] P. K. Tang and Y. H. Chew, "On the Modeling and Performance of Three Opportunistic Spectrum Access Schemes", IEEE Trans. Veh. Tech., vol. 59, no. 8, (2010).

[20] Y. Zhang, "Dynamic spectrum access in cognitive radio wireless networks", IEEE International Conference on Communications, Beijing, China, (2008).

[21] V. K. Tumuluru, P. Wang and D. Niyato, "Performance Analysis of Cognitive Radio Spectrum Access with Prioritized Traffic", IEEE International Conference on Communications, Kyoto, Japan, (2011).

[22] H. Huang, R.Y Yu, J. W. Wei, K. F. Xu and Y. S. Tao, "Design of the Wireless Video Monitor System", Journal of Harbin University of Science and Technology, vol. 19, no. 2, (2014).

[23] J. X. Qu, Y. Zhou and T. Y. Jiang, "Design and Simulation of Wireless Network MAC Protocol for Industrial Control System", Journal of Harbin University of Science and Technology, vol. 19 no. 3, (2014).

[24] Z. W. Chen and Q. Li, "Research and Realization of Video Multicast in the Wireless LAN", Jeunal of Harbin University of Science and Technology, vol. 14, no. 6, (2009).

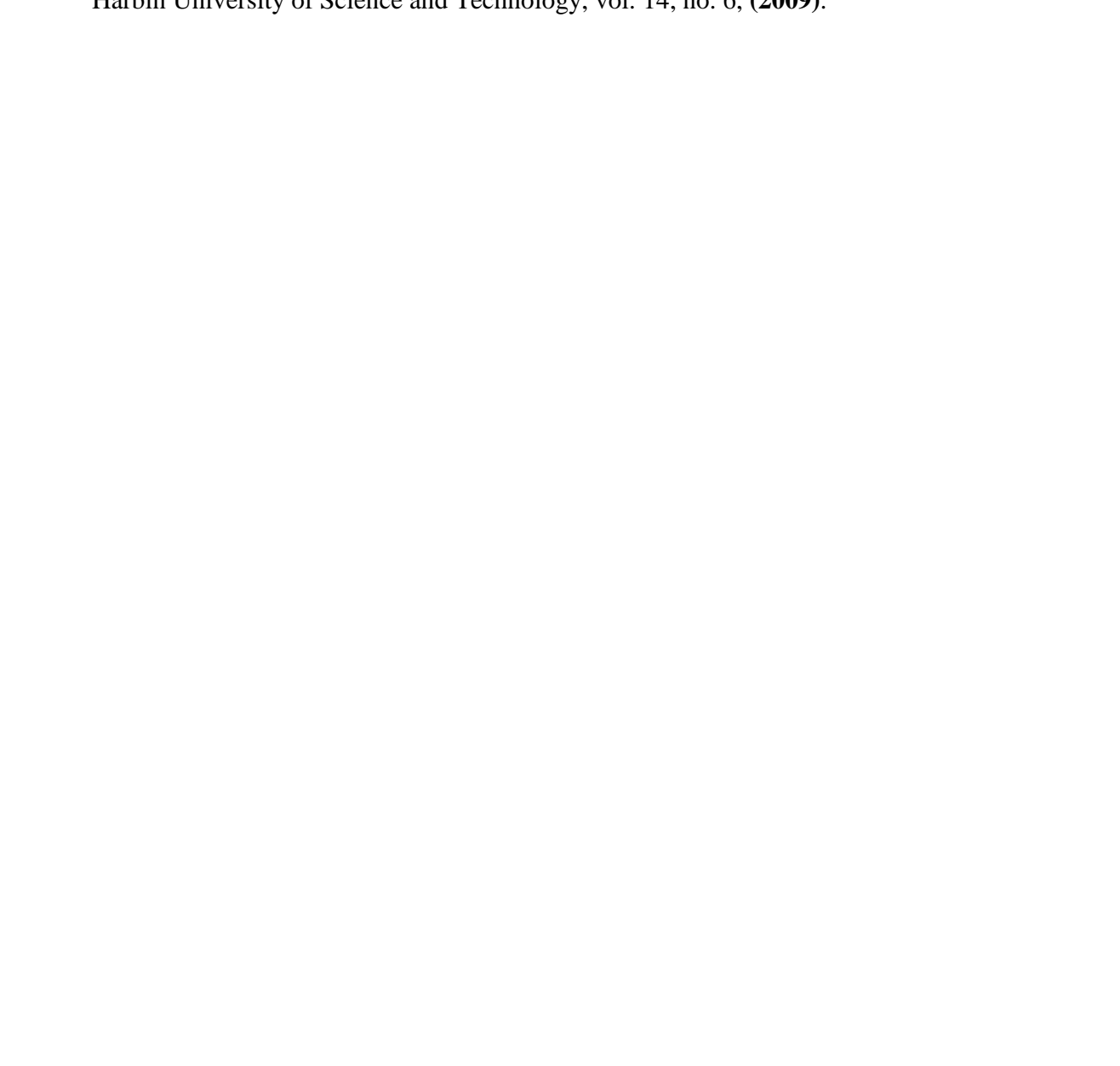


International Journal of Multimedia and Ubiquitous Engineering

Vol.11, No.9 (2016)

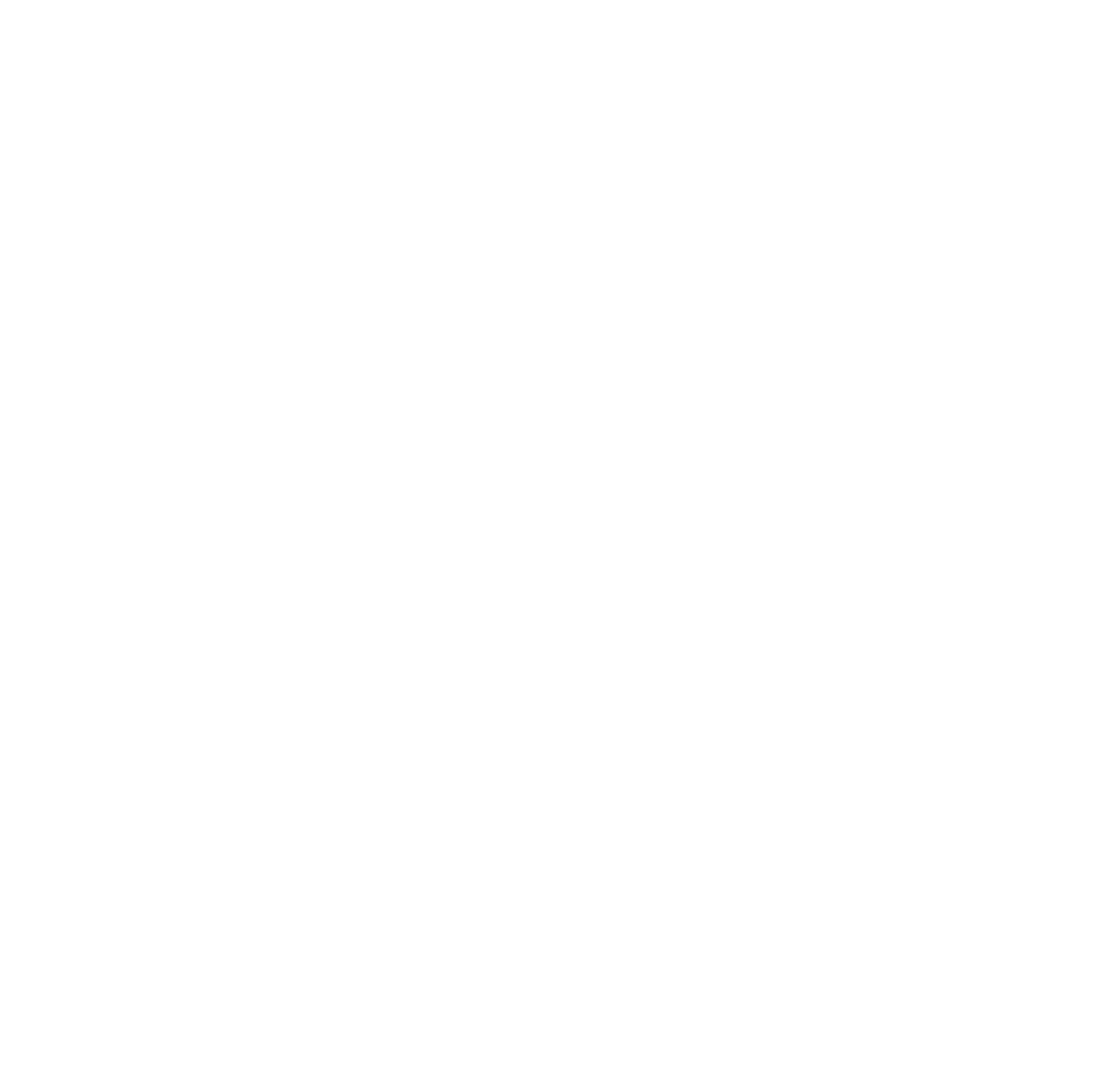

